\title{
ANTI-SARS NEWS
}

\section{Give Full Play the Role of Traditional Chinese Medicine to Prevent and Treat Severe Acute Respiratory Syndrome with Integrative Chinese and Western Medicine Approach}

Member of Political Bureau of Central Committee of CPC, Vice Premier of State Council concurrently Minister of Health, General Command of National Protecting and Treating Severe Acute Respiratory Syndrome ( SARS) Headquarters Vice premier WU Yi cordially met the wellknown TCM specialists, and held a symposium with them. Sixteen specialists including WANG Yong-yan, CHEN Ke-ji, LI Fu-ren, JIAO Shu-de, HE Pu-ren, LIU Zhi-ming and CHAO En-xiang as well as the leading officials of Ministry of Health. GAO Qiang, SHE Jing and ZHU Qingsheng, etc. attended the conference. The member of Using Integrative Chinese and Western Medicine (ICWM) in Treating SARS Project, WANG Rong-bin, etc. also attended the meeting. They have felt the solicitude of Party Central Committee and State Council for those medical professionals participated the rescuing and treating SARS and the expectations and encouragement to the TCM workers.

WU Yi listened to the opinions of all participants. The well-known veteran TCM doctors, JIAO Shu-de, HE Pu-ren and LIU Zhi-ming, etc. elucidated the principal approach of etiology, pathogenesis and syndrome differentiation from the TCM viewpoint. The Academicians WANG Yong-yan and $\mathrm{CHEN} \mathrm{Ke-ji} \mathrm{advocated} \mathrm{that} \mathrm{to} \mathrm{scientifi-}$ cally summarize the superiority of TCM in early treatment of SARS and its feasibility, to get hold of opportunity to promote the development of TCM cause. CHAO Enxiang, ZHANG Bing-hou and WANG Rong-bin reported the current situation of TCM in participating SARS treatment, as well as the basis of TCM treatment and its fit in point, namely: firmly grasp earlymiddle stage treatment, improve the symptoms, alleviate the adverse reaction of corticosteroids, reduce the complications and elevate the curative rate. At the same time they put forward some problems existed in current TCM treatment in treating SARS, such as whether the integrative treating program could reach the grassroot simultaneously, whether the doctors are encouraged to choose and use them, whether TCM specialty professionals role have been given their full play, etc.

Finally WU Yi summarized that she was greatly inspired by the participants' opinions, and pointed out that since SARS occurred in some districts in China, broad mass of TCM professionals actively devoted themselves to the prevention and treatment of SARS, particularly some aged TCM veterans, despite that their age is high, they actively ask for participation, which sufficiently display the lofty character of bearing the trouble of country, and solving the suffering of people, and the offering spirit of being fearless facing danger to heal the wounded and rescue the dying, which won the praise and respect of people mass. WU Yi expressed her heartfelt gratitude to over 1800 TCM practitioners in combating SARS in the frontline.

WU Yi stressed that TCM practitioner is an important force in combating SARS, one should sufficiently recognize the scientific value of TCM, actively utilize the resources of TCM, give full play the role of TCM practitioners, integrate the traditional Chinese and western medicine, to accomplish the common task of preventing and 
treating SARS. This conference indicated that the State Council has paid high attention to the TCM.

WU Yi pointed out that TCM has condensed the wisdom of Chinese nation, which is the science formed by the people of various nations in the long-term struggle $a^{-}$ gainst the diseases and through incessantly create, accumulate, enrich and develop, $\mathrm{a}^{-}$ bundant experiences have been accumulated in the prevention and treatment of major epidemic to formulate many unique theory and effective methods, it is the important constituents of our outstanding traditional culture. TCM not only contributes greatly to the prosperity of our Chinese nation, but also produces positive influences to the progress of world civilization. There is TCM medical care system which mainly consisted of TCM hospitals, with excellent TCM contingent, and complete TCM teaching system. After this SARS epidemic, the TCM specialists study and explore the SARS etiology and diagnosis-treatment regularity, actively apply TCM and ICWM approaches to treat and rescue the patient, and play positive role on it. These experiences are very valuable, it needs further summarize, study and popularize. At present, it is urgent to treat and rescue the patients at all costs, to lower the mortality, to ameliorate the frightened feeling of population.

WU Yi stressed that different departments of State Council and various level of governments should give full play of the role of TCM characteristics and superiority, encourage and support the TCM medical professionals to participate SARS prevention and treatment; various levels of health and TCM administrative should strengthen, organize, coordinate and guide, firmly get hold of manpower to launch the TCM scientific research to tackle the key problem, actively explore the TCM and ICWM approaches to prevent and treat SARS epidemic. Through practice and study, to raise the ability of TCM in preventing and treating SARS, to enrich and develop the academic and technologic level, to promote the TCM theory creation and technology creation, to keep the TCM vitality and activity. Integrative specialists and TCM specialists as well as specialist of other disciplines, and the inside of profession should strengthen the unity, mutual respect and coordination to each other, to strengthen the merit and offset the shortcomings of other side, to elevate the therapeutic efficiency of SARS treatment. WU Yi said, aged veteran TCM doctors are our precious fortune, it should both stress on playing their important role, earnestly listen to their opinions and suggestions, also create beneficial working and living condition of veteran TCM specialists.

As for the opinions of WU $\mathrm{Yi}$, it is now summarized as follows:

1. State Administration of TCM should sum up and systematize TCM treatment program of SARS, issued by Ministry of Health as basic treating methods.

2. In SARS designated hospital, special zones should be defined, TCM doctor should be responsible to treat with TCM therapy as principal approach. Middle and prime aged doctor as first line, aged veteran doctor as second line personnel.

3. ICWM should be insisted. ChinaJapan Friendship Hospital adopts ICWM in treating SARS, while Xuanwu Hospital would take western medicine in treating SARS for comparison.

4. Oriental Hospital in charge for a ward is a good suggestion, when problem occurs the leader of higher level would solve it.

5. The vitality of TCM should keep pace with the times, utilizing ICWM methods to rescue and treat critical severe $\mathrm{pa}^{-}$ tients.

(WANG Rong - bin) 